\title{
The ODDS Consortium Recommendations for Diagnosis of Optic Disc Drusen using Optical Coherence Tomography
}

Lasse Malmqvist, $\mathrm{MD}^{1}$, Lulu Bursztyn, Msc., $\mathrm{MD}^{2}$, Fiona Costello, $\mathrm{MD}$, $\mathrm{PhD}^{3}$, Kathleen Digre, $\mathrm{MD}^{4}$, J. Alexander Fraser, $\mathrm{MD}^{2,5}$, Clare Fraser, MMed. ${ }^{6}$, Bradley Katz, MD, PhD ${ }^{4}$, Mitchell Lawlor, FRANZCO, PhD ${ }^{6}$, Axel Petzold, $\mathrm{MD}, \mathrm{PhD}^{7}$, Patrick Sibony, $\mathrm{MD}^{8}$, Judith Warner, $\mathrm{MD}^{4}$, Marianne Wegener, $\mathrm{MD}^{1}$, Sui Wong, $\mathrm{MD}^{7}$, Steffen Hamann, MD, $\mathrm{PhD}^{1}$

\footnotetext{
${ }^{1}$ Department of Ophthalmology, Rigshospitalet, University of Copenhagen, Denmark

2 Department of Ophthalmology, Western University, London, ON, Canada

${ }^{3}$ Departments of Clinical Neurosciences and Surgery, University of Calgary, Calgary, Canada

${ }^{4}$ John A. Moran Eye Centre, University of Utah, Salt Lake City, UT, USA

${ }^{5}$ Department of Clinical Neurological Sciences, Western University, London, ON, Canada

${ }^{6}$ Sydney Eye Hospital, Sydney, NSW, Australia

${ }^{7}$ Moorfields Eye Hospital, London, United Kingdom and Neuro-ophthalmology Expertise Centre, VUmc, Amsterdam, $\mathrm{NL}$.
}

${ }^{8}$ Department of Ophthalmology, State University of New York at Stony Brook, Stony Brook, New York, USA

Collaborators:

Nitza Cohen, MD

Alison Crum, MD

Ruth Huna-Baron, MD

Hanna Leiba, MD

Hadas Stiebel-Kalish, MD

On behalf of the ODDS consortium

Running Title: The ODDS Consortium Recommendations for Diagnosis of Optic Disc Drusen

Word count: 1921

Keywords: Optic disc drusen; optic nerve head drusen; ODD; enhanced depth imaging optical coherence tomography; optical coherence tomography; recommendations; diagnosis.

All authors declare that they have no conflicts of interest.

\section{Corresponding author:}

Lasse Malmqvist

Department of Ophthalmology, Rigshospitalet

Nordre Ringvej 57, 2600 Glostrup, Denmark

Phone: 004538633340

Mail: lasse.malmqvist.larsen.01@regionh.dk 


\section{Abstract}

Background: A diagnosis of optic disc drusen (ODD) is important to exclude alternative lifethreatening diagnoses and to follow the slowly progressive visual field defects many ODD patients experience. The introduction of enhanced depth imaging optical coherence tomography (EDI-OCT) has made it possible to diagnose ODD reliably. There is however no consensus regarding the diagnosis of ODD using OCT. The purpose of this study was to develop a consensus recommendation for diagnosing ODD using OCT.

Methods: Based on the individual experiences of the involved researchers, ODD exercises, and discussions in the Optic Disc Drusen Studies (ODDS) consortium, a recommendation for OCT image acquisition and diagnosis of ODD was developed. The members of the ODDS consortium are either fellowship trained neuro-ophthalmologists with an interest in ODD, or researchers with a specific interest. Four standardization exercises were performed by the ODDS consortium with a focus on both image acquisition and diagnosis of ODD.

Results: Based on prior knowledge, and experiences from the exercises, the ODDS consortium reached a consensus regarding OCT acquisition and diagnosis of ODD. The recommendations from the ODDS consortium include scanning protocol, data selection, data analysis and nomenclature. Conclusions: The ODDS consortium recommendations for diagnosis of ODD using OCT are an important step for establishing a reliable and consistent diagnosis of ODD for both clinicians and researchers. 


\section{Introduction}

Optic disc drusen (ODD) are predominantly calcified deposits in the optic nerve head with an estimated prevalence of $2.4 \%$ (1). Because of methodological issues the published frequencies of patients with visual field defects have been highly variable, between $11.2 \%$ to $87 \%$ from representative large studies $(2,3)$. Visual field defects tend to progress slowly (4),. The condition is generally benign, with rare complications such as anterior ischemic optic neuropathy (AION) (5, 6). Confusion can arise because the frequently observed protrusion of the optic disc and blurring of the optic disc margin can be mistaken for papilledema (7-9). Separating one from the other is clinically important, as papilledema might be a manifestation of life-threatening increased intracranial pressure. Accurate diagnosis poses a challenge as buried ODD can be difficult to detect. Enhanced depth imaging optical coherence tomography (EDI-OCT) has proven to be more reliable in ODD diagnosis than B-scan ultrasound (10), which previously had been considered the gold standard (11).

The technique behind EDI-OCT was first described by Spaide et al. in 2008 (12). The resulting visualization and high resolution of the deeper layers of the optic nerve head has made it possible to reliably diagnose ODD. To date, however, there are conflicting descriptions of ODD morphology, and no agreement of a scanning protocol to best visualize ODD using OCT. The purpose of this paper was to develop recommendations for the OCT diagnosis of ODD. 


\section{Methods}

\section{The ODDS consortium}

The Optic Disc Drusen Studies (ODDS) consortium was established in 2015 as an international research alliance, with the aim to provide an international forum for optic disc drusen research to elucidate the mechanisms of ODD-related visual loss, and thereby provide potential treatment options. At the North American Neuro-Ophthalmology Society (NANOS) meeting in 2016, agreement was made between members of the consortium to develop consensus recommendations for the diagnosis of ODD. ODD morphology and a scanning protocol were discussed at this meeting and by further email correspondence.

\section{Patient Selection}

Different selections of EDI-OCT scans from 38 ODD patients attending the Department of Ophthalmology, Rigshospitalet, Denmark between November 2015 and January 2016 were used for the study exercises. All patients had been diagnosed using slit-lamp ophthalmoscopy, B-scan sonography or OCT. Only patients 18 years or older and patients without other ocular pathology visible on OCT were included. Further, EDI-OCT scans from healthy volunteers were obtained for use as control subjects. Examination of the patients had been approved by the local Scientific Ethics Committee (H-4, 2013-040) and conducted under the principles of the Helsinki declaration. Written informed consent was obtained from all patients prior to inclusion.

\section{EDI-OCT}

EDI-OCT (Spectralis OCT; Heidelberg Engineering, Heidelberg, Germany) was obtained in all patients using a preliminary ODDS consortium ODD scanning protocol (Table 1). All scans were obtained by the same experienced operator. The scanning procedure included a dense horizontal 
and vertical high resolution EDI-OCT scan of the optic nerve head. The scans were assessed and selected by the writing committee for the individual exercises using specialized software (Heidelberg Eye Explorer, version 1.7.0.0 and version 1.9.13.0, Heidelberg Engineering, Germany). Single B-scans were exported as individual image files, while volume scans were exported as E2E files. All files were exported with complete anonymization.

\section{ODD exercises}

A preliminary recommendation for ODD diagnosis and grading based on various discussions was developed by the writing committee and circulated together with the exercises.

\section{Exercise 1 - consensus approach to identify known ODD on EDI-OCT images: Eight} researchers participated in this exercise. A total of 22 EDI-OCT images, all with ODD, were included. The level of difficulty in diagnosing ODD varied between the images. The researchers were instructed to comment on localization, size and number of ODD and comment how they interpreted the picture.

Based on the replies, an updated preliminary recommendation to diagnose ODD was circulated among the researchers.

Exercise 2 - detection of ODD on EDI-OCT scans: Eight researchers participated in this exercise. A total of 20 EDI-OCT scans were included. The scans were a mix of eyes with and without ODD. The researchers were instructed to report presence or absence of ODD and to comment on the findings.

Exercise 3 - grading of ODD on EDI-OCT scans: Eight researchers participated in this exercise. A total of 10 scans were included. All scans were from eyes with known ODD. The researchers were instructed to grade ODD according to size and anatomical location.

Exercise 4-combining the knowledge: A total of 74 EDI-OCT scans were included. The scans were from eyes with ODD. The researchers were instructed to grade the scans according to size, 
structure and anatomical location. The researchers graded the same scans a second time, with a minimum of two weeks between the assessments. The anonymization and order of the scans was different between the two assessments. Ten researchers completed the first grading and eight researchers completed the second grading.

\section{Development of the ODDS recommendations}

The preliminary version of the ODDS recommendations for diagnosing ODD was based on the results, comments and discussions from the four ODD exercises. The writing committee generated a version of the protocol that was sent to all ODDS consortium members and later discussed and approved by consensus.

\section{Results}

\section{Recommended OCT protocol for diagnosing ODD}

The recommended ODDS consortium OCT protocol for diagnosis of optic disc drusen is seen in Table 1. The protocol is comprehensive but is designed to diagnose ODD with the best possible sensitivity and specificity. Furthermore, the protocol allows analysis of retinal and optic nerve head morphology for research purposes.

\section{ODDS recommendation for diagnosing ODD}

Based on the exercises and discussions a consensus regarding ODD morphology was reached. The morphology was confirmed in several cases where isolated autofluorescence or visible ODD on OCT en face view corresponded to underlying ODD visualized on OCT (Fig. 1). The OCT appearance of confirmed superficial ODD was extrapolated to identify buried ODD that could not be identified clinically. The ODDS consortium defined ODD as hyporeflective structures with a full or partial hyperreflective margin. The hyperreflective margin is often most prominent 
superiorly, and can be difficult to detect. The finding of a hyperreflective margin is useful for differentiating ODD from cysts.

Blood vessels imaged cross-sectionally sometimes mimic ODD but may be recognized by their "figure of eight configuration", vessel wall reflections and shadowing of underlying optic nerve head tissue (Fig. 2). Vessels imaged along the vessels longitudinal direction demonstrate a tri-layer profile with decreased intravascular reflectivity. Vessels imaged in more oblique planes often have a hyperdense "head" without visible "figure of eight configuration" but with underlying shadowing (Fig. 2). Furthermore, because our recommended OCT protocol allows one to "scroll" through parallel OCT slices to determine the 3D nature of optic nerve head structures, a review of several adjacent OCT slices can reveal the long tubular nature of a blood vessel, in contradistinction to the discrete "lump"-like nature of an ODD.

A peripapilary hyperreflective ovoid mass-like structure (PHOMS) was seen to surround the optic disc in 28 out of 38 ODD patients (Fig. 3). After discussing this morphological finding as a group, we decided not to include these structures as being ODD for several reasons: 1) They differ from our established ODD morphology by being hyperreflective without any sharp outer margin or hyporeflective core; 2 ) they often lie external to and surrounding large parts of the disc corresponding to funduscopically-recognized pseudopapilledema; 3) they exhibit no autofluorescence; 4) they are not visible on B-scan ultrasound despite their superficial location; 5) they are found in a large proportion of patients with true papilledema without evidence of underlying ODD; 6) Histopathology reports have described them as bended or herniated retinal nerve fibers, as an unspecific marker of axoplasmic stasis. Paton labelled them "lateral bulges" and by Cajal staining found that the structures were composed of nerve fibers (Fig.4). We therefore recommend that PHOMS are not diagnosed as ODD.

The ODDS consortium recommendation for diagnosing ODD using OCT is seen in Figure 5. As described previously, ODD always have a, hyporeflective core. Without a hyporeflective core other 
pathologies should be considered. ODD are often but not always seen with hyperreflective margins, most prominent superiorly. Sometimes collections of several smaller ODD coalesce to form ODD conglomerates that present with patchy internal reflectivity within a larger hyporeflective area. Hyperreflective horizontal lines are sometimes found in eyes with ODD or in otherwise healthy eyes in patients with unilateral ODD, and might possibly reflect early ODD changes. 


\section{Discussion}

This paper is the first to propose a recommendation for the use of OCT in diagnosing ODD. The purpose of the recommendation is to establish a reliable and consistent diagnosis of ODD using OCT for both clinicians and researchers. Better detection of ODD using OCT most likely will result in improved differentiation between buried ODD and papilledema. The distinguishing OCT features between the two entities have been widely investigated (13-19), with some studies focusing on retinal nerve fiber layer (RNFL) thickness differences $(13,14,16-18)$ and others on the so-called subretinal hyporeflective space $(15,16,18)$. The use of RNFL thickness to differentiate between the two was found to be clinically unreliable (17). This is supported by studies finding normal or increased RNFL thickness in patients with buried $\operatorname{ODD}(20,21)$. The use of the subretinal hyporeflective space to differentiate between the two is based on older OCT technology. With newer, more penetrating, OCT modalities such as EDI-OCT and swept source OCT (SS-OCT) there is no longer a subretinal hyporeflective space, but instead the entire optic nerve head is visible down to lamina cribrosa. This suggests that the hyporeflective space may be an imaging artifact.

The ODDS consortium reached a consensus to define ODD as hyporeflective structures with a full or partial hyperreflective margin. This is in agreement with most $(10,22-25)$ studies assessing ODD morphology using OCT. Some studies, however, have described ODD as hyperreflective structures with no hyporeflective core $(14,26,27)$ similar to what we in the ODDS consortium label PHOMS. These contradictory descriptions of ODD morphology using OCT have resulted in confusion in the diagnosis of ODD (28). After thoroughly reviewing and discussing, the ODDS consortium finds that there is no substantial evidence to diagnose PHOMS as ODD, as described above. However, there is a need to study PHOMS longitudinally to better understand how to classify these structures. Unless other evidence becomes available, the ODDS we recommend not to diagnose these structures as ODD. 
Another point of confusion is the hyperreflective horizontal lines often found in patients with ODD (10). Merchant et al. suggest the hyperreflective bands may themselves be an early sign of nascent ODD (10). This is supported in a study by Ghassibi et al., which found isolated hyperreflective bands in all eyes with ODD. Including these isolated bands in the definition of ODD resulted in an ODD prevalence of $14.6 \%$ among 130 clinically normal subjects (23). This prevalence is several times higher than ever reported and should be interpreted with caution. As is it still unclear whether these isolated hyperreflective bands in fact are ODD we recommend not diagnosing the hyperreflective horizontal lines as ODD until further evidence from longitudinal OCT studies emerges.

As described in this report there has been, until now, no consensus in the literature on how to diagnose ODD using OCT. The aim of our current ODDS Consortium recommendations is to help researchers and clinicians to diagnose ODD with high sensitivity and specificity. The recommendations are based on the individual experiences of the involved researchers, detailed study of the literature, four sets of ODD standardization exercises, and discussions in the ODDS consortium. By discussing the challenges of ODD diagnosis among experts from a consortium entirely dedicated to ODD, we aimed to establish consensus recommendations that can be used as a valid tool in ODD diagnostics.

There are several limitations that need to be addressed however. First of all, the recommendations are based on a selection of relevant studies but not on systematic reviews of literature. Our recommendations might change if new evidence becomes available. The recommendations are further based on the currently available OCT technology. Even though we are able to visualize the optic nerve head as far as down to lamina cribrosa there are still artifacts, noise and shadowing from overlying ODD and protruding optic discs, which provide challenges in interpretation. Rapid developments in OCT technology might improve the ability to visualize deeper structures in higher resolution without shadowing from overlying structures. This could 
change the way we diagnose ODD in the future. Ongoing reflections based on new evidence will likely refine the recommendations, and we regard our recommendations as a preliminary, but important, step towards improvement of ODD diagnosis using OCT. 
1. Friedman AH, Gartner S, Modi SS. Drusen of the optic disc. A retrospective study in cadaver eyes. The British journal of ophthalmology. 1975;59(8):413-21.

2. Erkkila H. Clinical appearance of optic disc drusen in childhood. Albrecht von Graefes Archiv fur klinische und experimentelle Ophthalmologie Albrecht von Graefe's archive for clinical and experimental ophthalmology. 1975;193(1):1-18.

3. Lorentzen SE. Drusen of the optic disk. A clinical and genetic study. Acta ophthalmologica. 1966:Suppl 90:1-180.

4. Malmqvist L, Lund-Andersen $\mathrm{H}$, Hamann S. Long-term evolution of superficial optic disc drusen. Acta Ophthalmol. 2016.

5. Purvin V, King R, Kawasaki A, Yee R. Anterior ischemic optic neuropathy in eyes with optic disc drusen. Archives of ophthalmology. 2004;122(1):48-53.

6. Nanji AA, Klein KS, Pelak VS, Repka MX. Nonarteritic anterior ischemic optic neuropathy in a child with optic disk drusen. Journal of AAPOS : the official publication of the American Association for Pediatric Ophthalmology and Strabismus / American Association for Pediatric Ophthalmology and Strabismus. 2012;16(2):207-9.

7. Braun A, Doniger SJ. Point-of-care ultrasonography for the identification of 2 children with optic disc drusen mimicking papilledema. Pediatr Emerg Care. 2014;30(7):505-7.

8. Sahin A, Cingu AK, Ari S, Cinar Y, Caca I. Bilateral optic disc drusen mimicking papilledema. Journal of clinical neurology. 2012;8(2):151-4.

9. $\quad$ Rott $D$, Leibowitz D. Optic nerve head drusen mimicking papilledema and malignant hypertension. Eur J Intern Med. 2009;20(5):e112-3.

10. Merchant KY, Su D, Park SC, Qayum S, Banik R, Liebmann JM, et al. Enhanced depth imaging optical coherence tomography of optic nerve head drusen. Ophthalmology. 2013;120(7):1409-14.

11. Kurz-Levin MM, Landau K. A comparison of imaging techniques for diagnosing drusen of the optic nerve head. Archives of ophthalmology. 1999;117(8):1045-9.

12. Spaide RF, Koizumi H, Pozzoni MC. Enhanced depth imaging spectral-domain optical coherence tomography. American journal of ophthalmology. 2008;146(4):496-500.

13. Lee KM, Woo SJ, Hwang JM. Differentiation of Optic Nerve Head Drusen and Optic Disc Edema with Spectral-Domain Optical Coherence Tomography. Ophthalmology. 2011;118(5):971-7.

14. Bassi ST, Mohana KP. Optical coherence tomography in papilledema and pseudopapilledema with and without optic nerve head drusen. Indian journal of ophthalmology. 2014;62(12):1146-51.

15. Johnson LN, DiehI ML, Hamm CW, Sommerville DN, Petroski GF. Differentiating optic disc edema from optic nerve head drusen on optical coherence tomography. Archives of ophthalmology. 2009;127(1):45-9.

16. Flores-Rodriguez P, Gili P, Martin-Rios MD. Sensitivity and specificity of time-domain and spectral-domain optical coherence tomography in differentiating optic nerve head drusen and optic disc oedema. Ophthalmic \& physiological optics : the journal of the British College of Ophthalmic Opticians. 2012;32(3):213-21.

17. Kulkarni KM, Pasol J, Rosa PR, Lam BL. Differentiating mild papilledema and buried optic nerve head drusen using spectral domain optical coherence tomography. Ophthalmology. 2014;121(4):95963.

18. Sarac O, Tasci YY, Gurdal C, Can I. Differentiation of optic disc edema from optic nerve head drusen with spectral-domain optical coherence tomography. Journal of neuro-ophthalmology : the official journal of the North American Neuro-Ophthalmology Society. 2012;32(3):207-11.

19. Katz BJ, Crum AV, Digre KB, Warner JEA. Optic Disc Edema and Optic Nerve Head Drusen. J Neuro-Ophthalmol. 2013;33(2):204-5. 
20. Gunes A, Demirci S, Tok L, Tok O, Demirci S. Evaluation of retinal nerve fiber layer thickness in Alzheimer disease using spectral-domain optical coherence tomography. Turk J Med Sci. 2015;45(5):1094-7.

21. Katz BJ, Pomerantz HD. Visual field defects and retinal nerve fiber layer defects are uncommon in patients with buried optic nerve drusen. J Invest Med. 2005;53(2):S398-S.

22. Sato T, Mrejen S, Spaide RF. Multimodal imaging of optic disc drusen. American journal of ophthalmology. 2013;156(2):275-82 e1.

23. Ghassibi MP, Chien JL, Abumasmah RK, Liebmann JM, Ritch R, Park SC. Optic Nerve Head Drusen Prevalence and Associated Factors in Clinically Normal Subjects Measured Using Optical Coherence Tomography. Ophthalmology. 2017;124(3):320-5.

24. Slotnick S, Sherman J. Buried disc drusen have hypo-reflective appearance on SD-OCT. Optometry and vision science : official publication of the American Academy of Optometry. 2012;89(5):E704-8.

25. Yi K, Mujat M, Sun W, Burnes D, Latina MA, Lin DT, et al. Imaging of optic nerve head drusen: improvements with spectral domain optical coherence tomography. Journal of glaucoma. 2009;18(5):3738.

26. Lee KM, Woo SJ, Hwang JM. Morphologic characteristics of optic nerve head drusen on spectral-domain optical coherence tomography. American journal of ophthalmology. 2013;155(6):1139-47 e1.

27. Lee KM, Hwang JM, Woo SJ. Optic disc drusen associated with optic nerve tumors. Optometry and vision science : official publication of the American Academy of Optometry. 2015;92(4 Suppl 1):S67-75.

28. Slotnick S, Sherman J. Disc drusen. Ophthalmology. 2012;119(3):652; author reply -3 e1. 29. Paton L, Holmes G. The Pathology of Pailloedema: A Historical Study of Sixty Eyes. Brain. 1911;33(4):389-432.

30. Tso MO. Pathology and pathogenesis of drusen of the optic nervehead. Ophthalmology. 1981;88(10):1066-80. 
Table 1. Advised OCT protocol for diagnosis of optic disc drusen

$1 \quad$ Before scanning

2 Acquisition

3

Dense optic nerve head scan

4

Radial optic nerve head scan

5

Peripapillary scan

6

\section{Macula scan}

Ensure optimal conditions

Dilate pupils before examination

b Measure corneal curvature and refraction for later transverse magnification adjustment (to ensure accurate measurements) *

\section{Visualize deeper structures}

Use SD-OCT in EDI mode or SS-OCT

b If no SD-OCT or SS-OCT is available, adjust the distance from the OCT apparatus to the eye to get an inverted view of the optic nerve head for better visualization of deeper structures Type in corneal curvature value and refraction in the operator system*

\section{Identification, quantification and classification of ODD}

Use EDI mode or invert scan

b Select high resolution acquisition if possible

c Center a scan area of only $15 \times 10$ degrees over the optic disc

d Scan with 97 sections in that area (30 $\mu \mathrm{m}$ between each scan)

e $\quad$ Average at least 30 frames

f Perform the volume scan in both horizontal and vertical directions

a Use EDI mode or invert scan

b Select 20 degree 6 line radial scan (star pattern scan)

c Center scan at optic disc

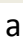

\section{Evaluation of RNFL thickness}

Deselect EDI mode (if on)

b Select 12 degree peripapillary scan (circle scan)

C Center scan at optic disc

\section{Exclude macular pathology}

a Deselect EDI mode (if on)

b Center a scan area of $20 \times 20$ degrees over macula.

c Scan with at least 25 sections ( $240 \mu \mathrm{m}$ between each scan)

d Average at least 9 frames

$7 \quad$ Autofluorescence

\section{Identification of autofluorescence positive ODD}

Center the scan at the optic disc

Average 100 frames

Abbreviations: OCT, optical coherence tomography; SD-OCT spectral domain optical coherence tomography; EDI, enhanced depth imaging; SS-OCT, swept source optical coherence tomography; ODD, optic disc drusen; RNFL, retinal nerve fiber layer. * Only applicable when using Heidelberg OCT. 


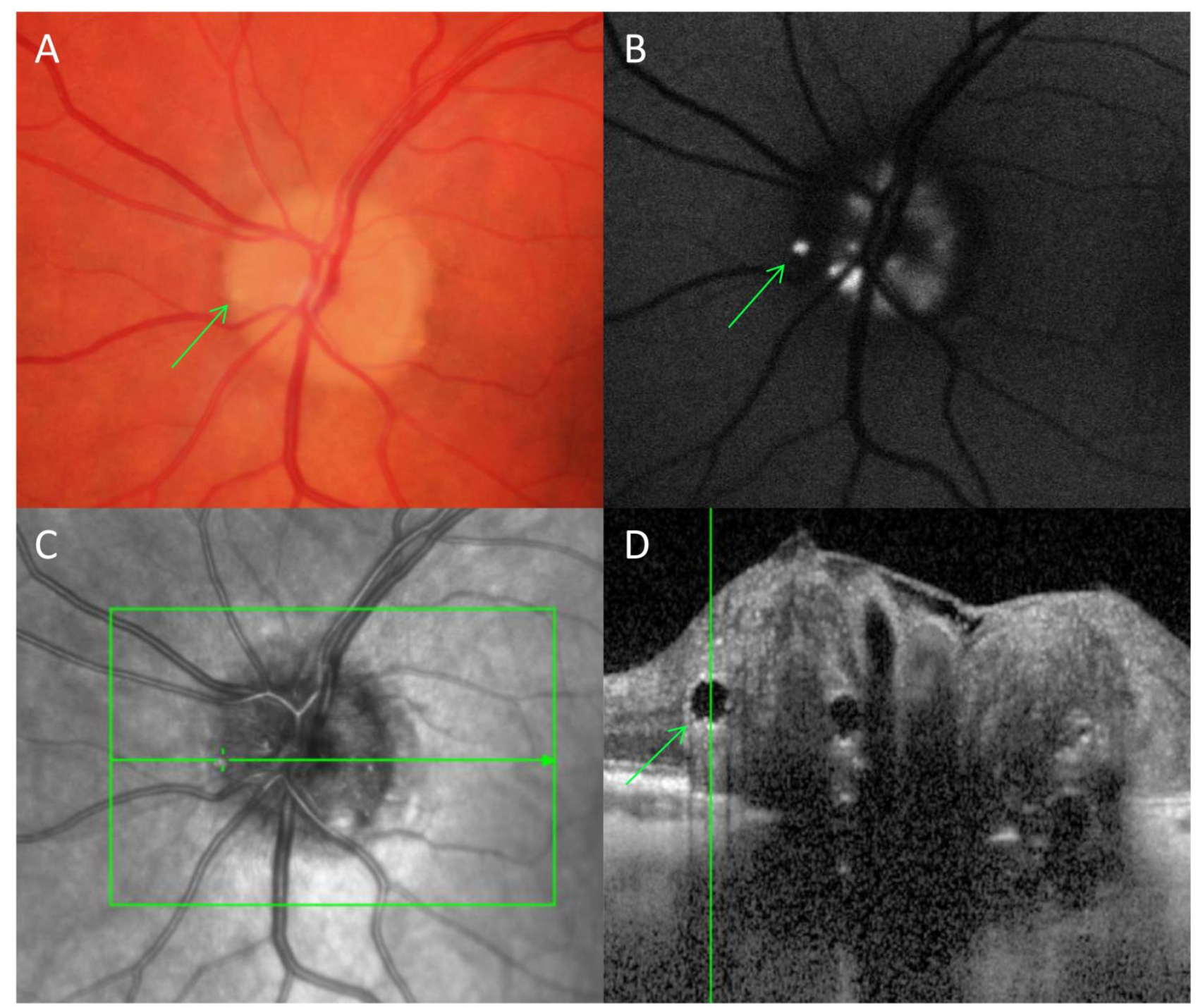

Figure 1. A, Fundus photography of an optic disc with optic disc drusen (ODD). An isolated ODD is seen nasally (green arrow). B, Fundus autofluorescence of the same disc. An isolated sharply demarcated area of autofluorescence (green arrow) has been detected as a visible calcified ODD. The larger and more diffuse signal corresponds to larger and deeper ODD. C, En face infrared view of the same optic disc. Green square is representing scan area and green horizontal arrow is visualizing scan direction and level. A visible ODD (green arrow) is seen corresponding to the visible ODD on fundus photography and autofluorescence. D, OCT Bscan of the same optic disc with the green line corresponding to the horizontal green arrow in C. An isolated ODD (green arrow) is seen to correspond to the fundus photography, fundus autofluorescence and en face view. The ODD is hyporeflective with a hyperreflective margin. Other deeper ODD are also seen corresponding to the diffuse signal on fundus autofluorescence. 
Figure 2. Optic disc drusen and vessel configuration on OCT. A, B, En face infrared view and optical coherence tomography scan of optic disc with green square representing scan area and green horizontal arrow visualizing scan direction and level. Asterisk indicates cross-sectionally recorded vessels. White arrowheads indicate vessels recorded in an oblique angle while long white arrows indicate vessels recorded along the vessel direction recognized by their tri-layer profile. A large hyporeflective ODD with superior hyperreflective margin is seen at the level of Bruch's membrane opening. C, Close-up visualization of vessels exhibiting figure of eight configuration. 
Figure 3. En face infrared overview and EDI-OCT from an optic disc drusen (ODD) patient with ODD-related pseudopapilledema (AD) and an ODD patient without pseudopapilledema (E-F). A,B, A peripapillary hyperreflective ovoid mass-like structure (PHOMS) is seen mainly temporally (white arrows). The patient has massive optic disc protrusion caused by optic disc drusen. Green square represents scan area and green lines the location of B-scan. C,D, Segmented PHOMS (red overlay) in the same patient. The structure is seen to circumscribe a large part of the optic disc causing the blurred margins in the en face view. E,F, Optic nerve head scan from an ODD patient without blurred margins or optic disc protrusion. No PHOMS are visible in this scan. White asterisk indicates an ODD. Red arrow indicates blood vessels. Yellow arrow indicates ODD conglomerates. 

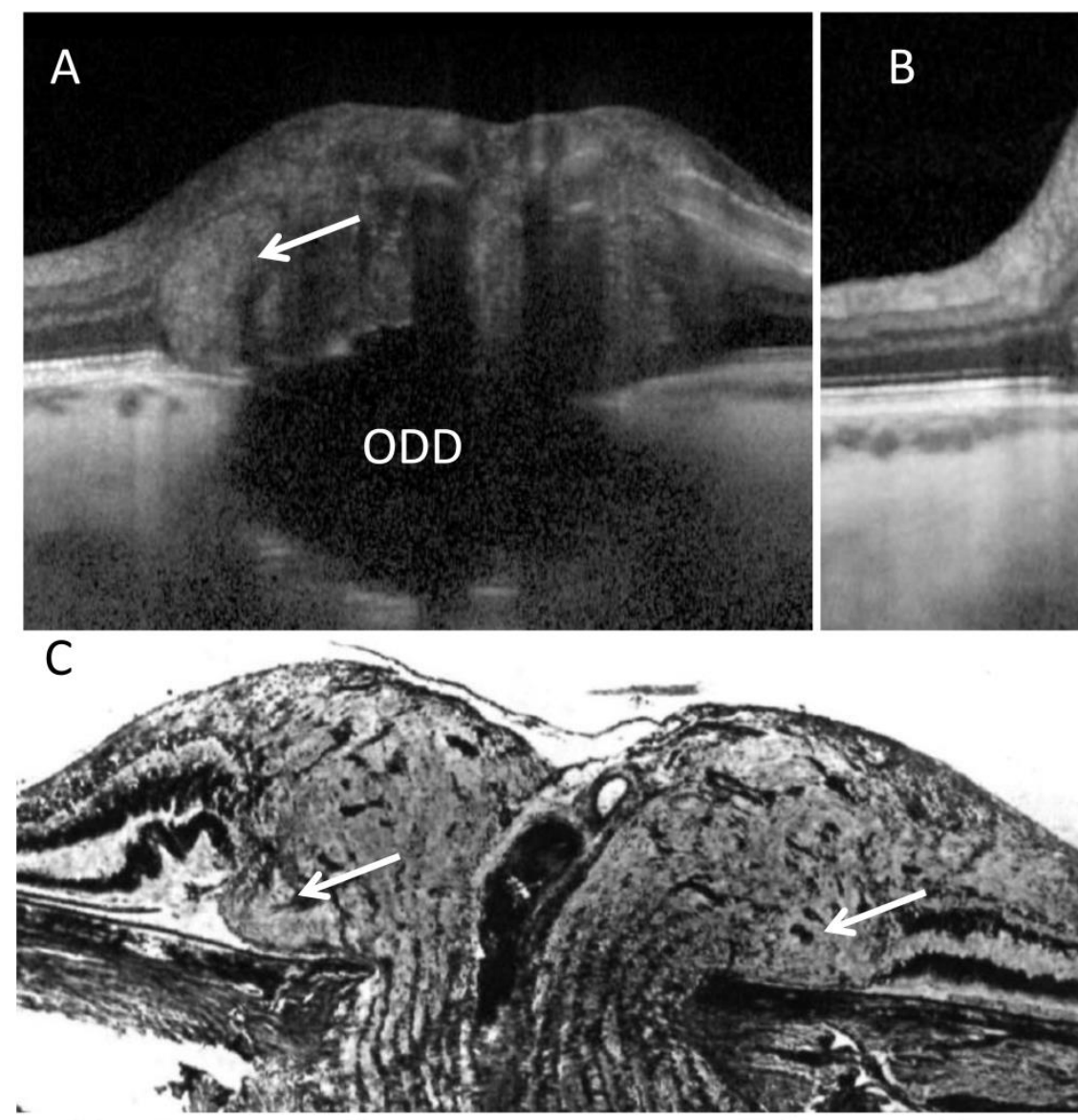

\section{D}
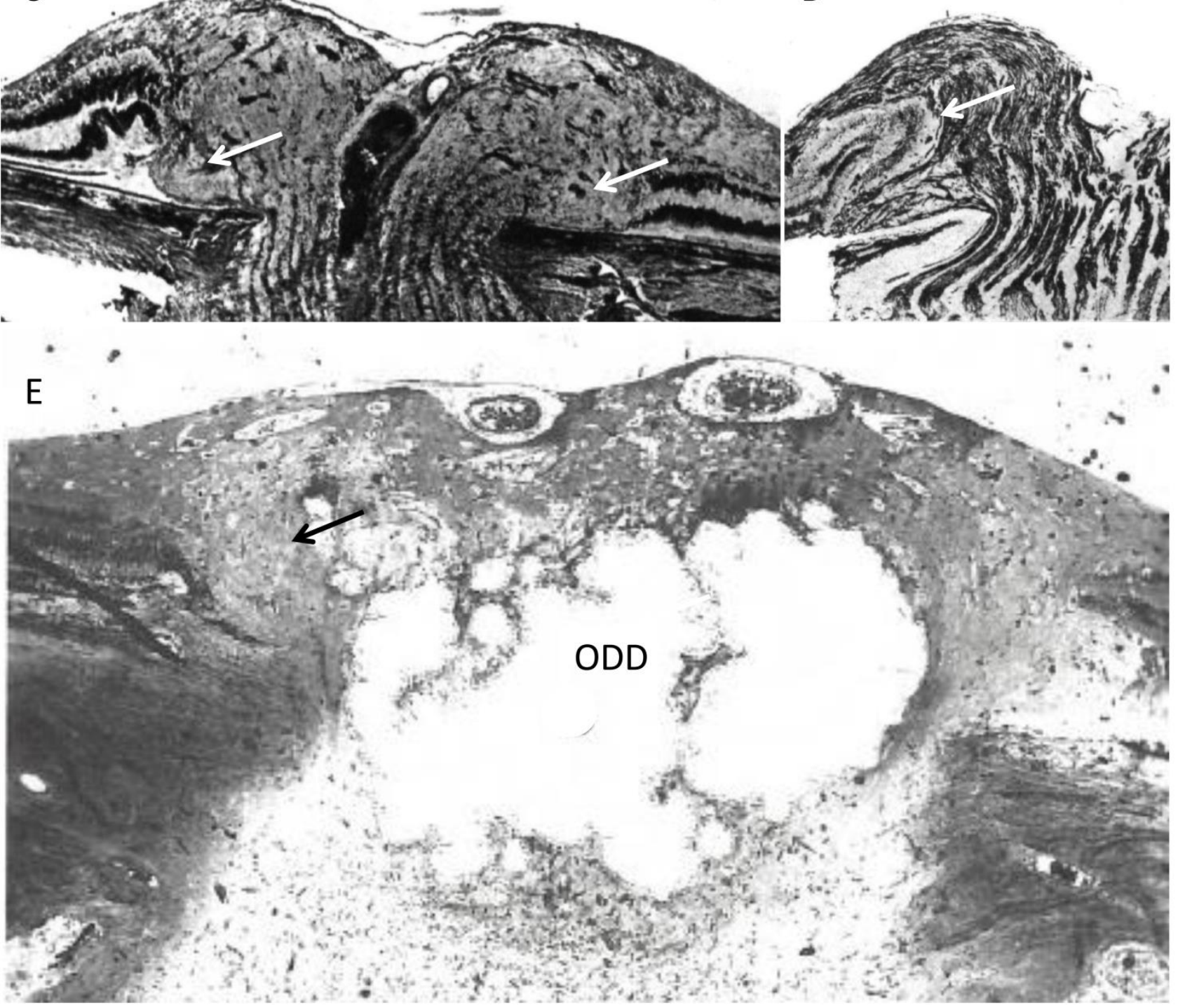

Figure 4. A, Enhanced depth imaging optical coherence tomography of a 28-year-old patient with optic disc drusen (ODD) showing a peripapillary hyperreflective ovoid mass like structure (PHOMS) (white arrow). B, A similar PHOMS (white arrow) in 35-year-old patient with idiopathic intracranial hypertension $(\mathrm{IIH})$ ) with the same morphological characteristics as the structure seen in the ODD patient. A shadow from an overlaying vessel is traversing the structure. C, Histopathologic section of another patient with papilledema showing lateral bulging (white arrows) of nerve fibres corresponding to PHOMS seen by OCT. D, when section is stained by Cajal's method the course of the peripheral bundle is compressed until at the level above Bruch's membrane where after it forms the mass of the lateral bulge. Reprinted from Paton \& Holmes (29) with permission from Oxford University Press. E, Histopathologic section of a patient with ODD showing lateral bulging of nerve fibres corresponding to PHOMS seen in eyes with ODD using OCT. Reprinted from Tso (30) with permission from Elsevier. 


\section{Diagnosing optic disc drusen using optical coherence tomography}

- ODD are always located above lamina cribrosa

- ODD always have a signal-poor core

- ODD are often seen with a hyperreflective margin, most prominent superiorly

- ODD are sometimes seen as conglomerates of smaller ODD with internal reflectivity within the signal-poor core

- Hyperreflective horizontal lines might represent early ODD but should not be diagnosed as ODD

- The classification of peripapillary hyperreflective ovoid mass-like structures (PHOMS) will require further study

Figure 5. Optic disc drusen (ODD) morphology on enhanced depth imaging optical coherence tomography (EDI-OCT). ODD are seen as signal poor structures with a partial hyperreflective margin. Peripapillary hyperreflective mass-like structure is surrounding the disc. Internal reflectivity represents the fusion of two ODD. Hyperreflective horizontal lines might be early ODD or, in this case because of the deep location, signal from lamina cribrosa. Peripapillary hyperreflective ovoid mass-like structures (PHOMS) circumscribe the disc. 